\title{
Restart Algorithm of Infertility and IVF Procedures in Patients with Desire of Baby Throughout COVID-19 Pandemic
}

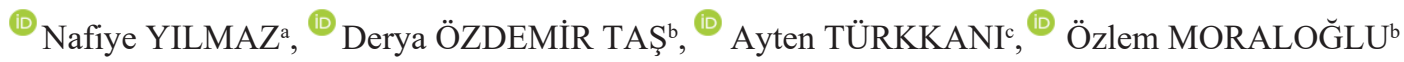 \\ ${ }^{a}$ University of Health Science, Ankara City Hospital, Department of Obstetrics and Gynecology, Ankara, TURKEY \\ ${ }^{\mathrm{b}}$ Ankara City Hospital, Department of Obstetrics and Gynecology, Ankara, TURKEY \\ ${ }^{c}$ University of Health Science, Gülhane Faculty of Medicine, Department of Histology and Embriology, Ankara, TURKEY
}

\begin{abstract}
COVID-19 infection, which was first detected in Wuhan region of China, spread rapidly in a short time, caused a very serious epidemic and was declared as a pandemic. This manuscript has been prepared as a recommendation for centers planning to restart assisted reproductive technology treatments to protect both patients and health personnel. To carry out the treatment, surgery and in vitro fertilization for patients who are infertile and are willing to have a child should not be victims of time loss during the COVID-19 pandemic. Patients before or after infertility treatment are evaluated by healthcare personnel using personal protective equipment in the triage section for the symptoms and risk factors defined in the COVID-19 Infection Guideline of the Ministry of Health. Body fluids (blood, follicular fluid, semen, etc.) of the patients should be considered as potentially infectious. Samples from patients infected with COVID-19 are recommended to be used in the same way as tissues from patients who are seropositive for other infectious diseases (HIV, Hepatitis). Infertility treatment and IVF procedures should be evaluated multidisciplinary and managed according to the COVID-19 guidelines in patients with infertility especially in poor ovarian reserve, severe male factor infertility, advanced age and also urgent fertility preservation condition.
\end{abstract}

Keywords: COVID-19; pandemic; infertility

The new type of Coronavirus infection COVID19 (SARS-CoV-2), which was first detected in Wuhan region of China in December 2019, spread rapidly in a short time and caused a very serious epidemic. The World Health Organization declared this infection, which affects the whole world, as a new type of Coronavirus pandemic on 11 March $2020{ }^{1}$

COVID-19 infection has very fast transmission through droplet with direct contact, coughing, sneezing. Although the incubation period of the disease is 3-7 days, it can be extended up to 14 days. While $80 \%$ of the patients have the disease without any symptoms, approximately $15 \%$ of them present with fever, cough and shortness of breath, pneumonia or difficulty in breathing . Approximately $5 \%$ of patients need intensive care unit and respiratory support units due to 'Severe Acute Respiratory Syndrome' and multiorgan failure. The pathophysiology, epidemiology and transmission of the disease are not yet completely understood, and a certain antiviral treatment option and vaccine are not yet available. ${ }^{2}$

In order to control this disease, which affects the worldwide and health systems; initially, protection methods should be given matter, and working conditions of the health system and health personnel should be reorganized in this process.

Preventive measures of major sinificance should be the limitation of the mobility of especially asymptomatic patients, which consist of almost $80 \%$ of whole patient population, ensuring isolation, especially respecting hand hygiene, preventing surface contamination, and ensuring the use of personal pro-

Correspondence: Nafiye YILMAZ

University of Health Science, Ankara City Hospital, Department of Obstetrics and Gynecology, Ankara, TURKEY

E-mail: nafiyekarakas@gmail.com

Peer review under responsibility of Journal of Clinical Obstetrics \& Gynecology.

Received: 18 May 2020 Accepted: 31 May 2020 Available online: 11 Jun 2020

2619-9467 / Copyright @ 2020 by Türkiye Klinikleri. This is an open

access article under the CC BY-NC-ND license (http://creativecommons.org/licenses/by-nc-nd/4.0/). 
tective equipment and isolation of health personnel who come into contact with the exact or suspicious cases. Despite all of these protective measures, it is essential to use both health care facilities and personnel more efficiently, due to the potential of rapid increase number of patients who need to be hospitalized or need intensive care and respiratory support units, because of the rapid transmission property of the disease. Therefore, the number of personnel working and mobility to be provided in hospitals should also be minimized. For this purpose, elective procedures should be delayed as long as this situation remains uncertain. All over the world and as well as our country, elective procedures and treatments have been stopped in line with the recommendations of health authorities and associations (CDC, WHO, ASRM, ESHRE, RCOG, ACOG, ... etc). However, it is not known how long the pandemic will continue and whether there will be a second wave. ${ }^{3,4}$ In this uncertainty, taking into account the possibility of long lasting process, alternative algorithms for health care fascilities should be prepared for the transition period where the infection density is not extreme until normal conditions are established for patients who should not be victims of this process in terms of time.

COVID-19 pandemics required new working reorganizations in healthcare services worldwide. Throughout this reorganization, some procedures and treatment applications in infertile patients also had to be rearranged. This guide has been prepared to take the necessary measures to protect both patients and health personnel. To carry out the surgery and in vitro fertilization treatment procedures for patients who are infertile and have a desire to have a child should not be victims of time loss during the COVID-19 pandemic.

\section{INSTRUCTIONS FOR PATIENTS}

Management of the patients who are admitted for examination and treatment should be resumed according to the algorithm (Figure 1). The patients who admitted to the health care facility with child request during the COVID-19 pandemic process are evaluated by the health personnel using personal protective equipment (PPE) in the triage section in terms of infection symptoms and risk factors defined in the COVID-19 Infection Guide of the Ministry of Health, and the risk group is defined by filling the risk assessment form (Table 1, Table 2). ${ }^{3}$

\section{Risk-free case}

2. Suspicious case

\section{Confirmed case}

Firstly the patients who have no risk or low risk and have no symptoms of disease are accepted for examination. The patient and the partner if any, are informed about COVID-19 infection and their treatment is completed after obtaining their consent. In this process, since the COVID-19 infection can be asymptomatic at a rate of $80 \%$, absolute use of PPE

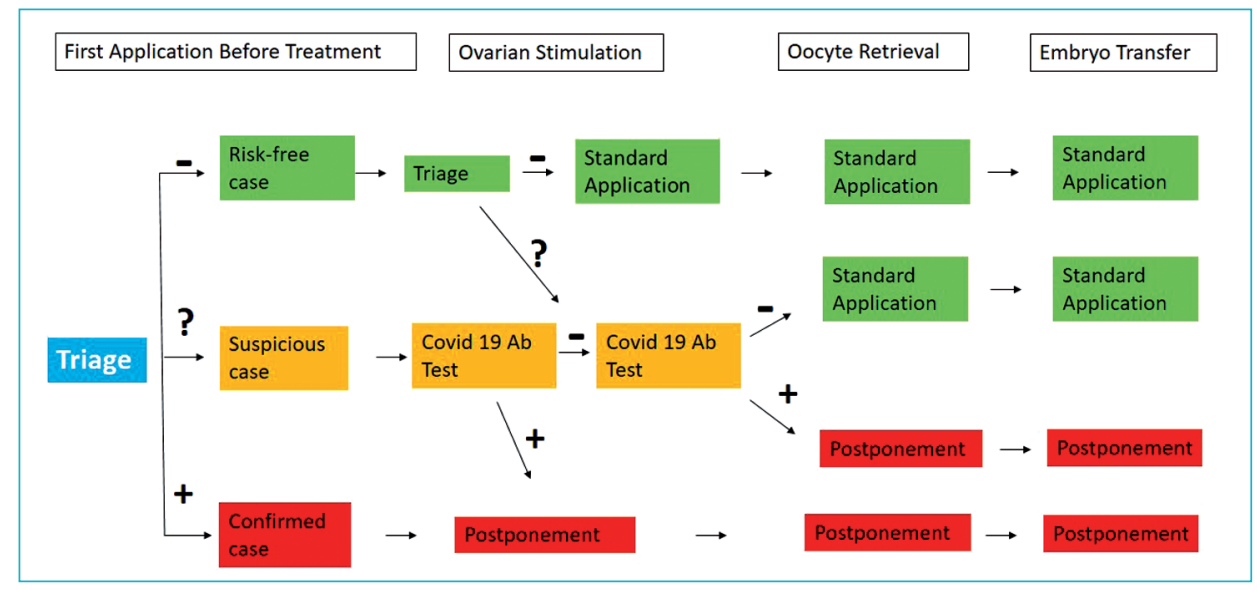

FIGURE 1: Infertility and IVF patients' management algorithm under COVID-19 pandemic. 
TABLE 1: Assessment of healthcare professional's contact status with COVID-19 patient.

\begin{tabular}{|lll|}
\hline \multirow{2}{*}{$\begin{array}{l}\text { Intense contact with COVID-19 } \\
\text { patient wearing a medical mask }\end{array}$} & Use of Personal Protective Equipment (PPE) of Healthcare Professionals & Contact Risk \\
& No medical mask or N95 mask or use of medical mask in case of & Medium \\
& an N95 mask indication & Low \\
& No eye protection & Low \\
& No gloves or gown used & No Risk \\
& All PPE used appropriately & High \\
Intense contact with & No medical or N95 masks & Medium \\
COVID-19 patient without medical mask & Use of medical masks in case of N95 mask indication & Medium \\
& No eye protection & Low \\
& No gloves or gown used & No Risk \\
& All PPE used appropriately &
\end{tabular}

TABLE 2: Outpatient triage inquiry guide.

\section{Outpatient Triage Inquiry Guide}

Triage is performed by a healthcare professional dressed in accordance with the COVID-19 case algorithm (gown, medical mask, face shield, or goggles).

$\begin{array}{lcc}\text { Do you have a fever or a fever history? } & \text { YES } & \text { NO } \\ \text { Do you have a cough? } & \text { YES } & \text { NO } \\ \text { Do you have breathing difficulties or respiratory problems? } & \text { YES } & \text { NO }\end{array}$

If the answer to any of the above questions is YES, the PATIENT MASK IS WORN and directed to the area reserved for COVID-19.

If the answer to all of the above questions is NO, the following questions are asked.

$\begin{array}{lll}\text { Have you been abroad in the last } 14 \text { days? } & \text { YES } & \text { NO } \\ \text { Has households come from abroad in the last 14 days? } & \text { YES } & \text { NO } \\ \text { Has any of your relatives been hospitalized for respiratory disease in the last 14 days? } & \text { YES } & \text { NO } \\ \text { Has any of your relatives diagnosed with COVID-19 disease in the last 14 days? } & \text { YES } & \text { NO }\end{array}$

If the answer to any of the questions is YES, the PATIENT MASK IS WORN and directed to the area reserved for COVID-19.

If the answer to all of the above questions is NO, it is considered as low risk for COVID-19 and directed to the relevant department for consideration in the direction of its complaint.

is important for both the patient and the health personnel. ${ }^{3}$

After risk evaluation, patients with risk factors and showing symptoms of disease are not admitted to the IVF treatment program and their treatment is delayed until the test results are clear. It is ensured that the patient is directed to the relevant COVID-19 monitoring center with a report and should use absolute PPE.

The condition and planning of the treatment process of patients with poor ovarian reserve, serious male factor or fertility preservation, who are concerned about loss of time in terms of infertility and IVF procedures should be evaluated in line with the recommendations of the guidelines considering the general health status and priorities of the patients. Applications should be updated according to the suggestions that will occur as a result of reporting the data. ${ }^{3,4}$

Secondly, patients should be evaluated again in terms of infection symptoms and risk factors in the triage section during the ovarian stimulation. Patients who do not have a risk stimulation treatment should be continued. Patients with risk factors or patients that show symptoms during their treatment after risk assessment are provided to use absolute PPE until the test results are cleared, and are directed to the relevant COVID-19 monitoring center. The patient 
should be evaluated by the infectious diseases specialist and obstetrician after the tests are completed. It is planned to follow up according to the decision of infectious diseases specialist until the test result is clear. The condition and planning of the treatment process of patients with poor ovarian reserve, serious male factor or fertility preservation, who are concerned about loss of time in terms of infertility and IVF procedures, and the planning of the treatment process should be evaluated in line with the recommendations of the guidelines considering the general health status and priorities of the mother or father. Applications should be updated according to the suggestions that will occur as a result of reporting the data. $^{3,4}$

Surgical Procedure: For the patient decided to operate, the personnel is informed according to the previously determined work list.

If it is decided to perform the operation of the suspicious/infected patient, the operation and procedures of the suspicious/infected patient are designed in a different time frame from other patients and to minimize contact with other people. Entrance area of the operating room and treatment room should be separated for suspicious/infected patients. A separate operating room should be defined for the operations.

The surgical team wears a heading and a mask when entering the operating room, and then in the protective equipment dressing room; wears a N95 mask, workwear and foot protection for procedures involving aerolization according to the determined risk.

The surgical team wearing protective equipment wears double sterile gloves and a sterile gown to prevent contamination during removal after hand washing according to the surgical hand washing instructions. The embryology laboratory team also wears PPE and double sterile gloves and gowns after washing hands according to the surgical hand washing instructions.

During this process, the number of healthcare personnel to be present in the procedure room should be kept to a minimum, and if possible, the procedure should be completed in the negative pressure room, and entry and exit should be limited during the pro- cedure (1 obstetrician, 1 instrument nurse, 1 anesthesiologist, 1 anesthesia technician, 1 assistant personnel and 1 embryologist and/or biologist). During contact with the infected patient for operation, PPE should be used by all healthcare personnel and attention should be paid to the use of PPE for the patient.

Anesthesia Procedure: Anesthesia team dressed in protective equipment performs anesthesia. To protect from the patient's respiratory secretions, anesthesia team places protective barrier from the patien's chest area and works with the closed system. The surgical team starts the patient's surgery after the anesthesia procedure. ${ }^{3,4}$

Embryology Laboratory Procedure: IVF procedures and surgery of the patient whose treatment decision has been made is completed by using the necessary personal protective equipment (PPE) defined in the COVID-19 Infection Guideline of the Ministry of Health for all healthcare personnel. All body fluids (blood, follicular fluid, semen, etc.) should be potentially infectious. ${ }^{3}$ There is a risk of contamination of diseases from these samples to staff and other patients' biological materials (cross contamination). The embryology laboratory team stuff must comply with the required PPE use and asepsis techniques. Treatment of suspicious/infected patients should only be performed in in vitro fertilization (IVF) laboratories with special fields and equipment to ensure appropriate safety measures. Alternatively, in the treatment of patients, specific time periods can be separated to thoroughly disinfect work areas and equipment following the processing of biological samples. The embryology and andrology laboratories should have the minimum number of staff required to perform gamet and embryo safety, performance and all quality control activities of emergency cases. The immediate interaction of the laboratory team with patients should be minimized.

Sperm Washing Process in Andrology Laboratory: Sample should be prepared in vertical laminar flow cabinets suitable for biological samples processing. If possible, a separate incubator should be used. Disposable consumables should be disposed of immediately after use in appropriate waste boxs, elimination of potentially contaminated material to 
prevent exposure of laboratory workers and other personnel, elimination of virus positive material in separate containers, labeling and disposal in accordance with biosafety rules. Needles, glassware and other sharp objects should be handled with extreme care and thrown into the cutter waste containers. All surfaces must be disinfected after treatment with disinfectants with proven suitability and effectiveness for IVF laboratories. The manufacturer's recommendations should be followed, including dilution, contact time and safe use of the disinfectant. This cleaning should be done immediately after use and before putting the equipment back into the general service. During all applications, the laboratory team should pay attention to the use of PPE and all operations (except for intracytoplasmic sperm injection application) should be done in vertical laminar flow cabinets. After each treatment, the surfaces should be cleaned with distilled water. Disposable consumables and material with potential contamination risk should be disposed of in separate waste boxes immediately after use, and labeled and disposed of in accordance with biosafety rules. Glass pipettes and materials should be handled with extreme care and thrown into the separate cutter waste containers. If possible, a separate incubator should be used for embryos and gametes of infected patients. All surfaces must be disinfected after treatment with disinfectants with proven suitability and effectiveness for IVF laboratories. ${ }^{5,6}$

In tissue preservation, it is not known whether cross-contamination will occur between samples of patients infected with Coronovirus (oocyte, sperm, embryo, ovarian, and testicular tissue) and non-infected patients. However, until otherwise approved, samples from patients infected with Coronavirus are suggested to be used in the same way as tissues from patients who are viral-positive for other infectious diseases (HIV, Hepatitis). It is suggested to use separate cryo tanks. Closed system straws should be used. Although the data on the transmisson of COVID-19 with liquid nitrogen is unclear, personnel used in the embryology laboratory should use the necessary PPE during procedures involved to cryo tanks and during contact with the infected patient. ${ }^{5,6}$

Disinfection of infected rooms and operating rooms used in IVF cycles should follow the advice in the COVID-19 Guidelines of the Ministry of Health.

\section{CONCLUSION}

After evaluating the case data obtained during the COVID-19 pandemic process and according to novel evidence, the approach in pregnancy interaction and reproductive therapy applications should be updated as well as other practices and approaches.

\section{Source of Finance}

During this study, no financial or spiritual support was received neither from any pharmaceutical company that has a direct connection with the research subject, nor from a company that provides or produces medical instruments and materials which may negatively affect the evaluation process of this study.

\section{Conflict of Interest}

No conflicts of interest between the authors and / or family members of the scientific and medical committee members or members of the potential conflicts of interest, counseling, expertise, working conditions, share holding and similar situations in any firm.

\section{Authorship Contributions}

Idea/Concept: Nafiye Yllmaz, Özlem Moraloğlu; Design: Nafiye Yılmaz, Ayten Türkkanl; Control/Supervision: Özlem Moraloğlu; Literature Review: Derya Özdemir Taş; Writing the Article: Nafiye Yllmaz, Ayten Türkkanl; Critical Review: Özlem Moraloğlu.

\section{REFERENCES}

1. WHO. WHO Director-General's opening remarks at the media briefing on COVID-19 -29 June. 2020. [Link]

2. The National Institutes of Health. Coronavirus disease 2019 (COVID-19) treatment guidelines. 2020. [Link]

3. T.C. Sağlık Bakanlığı Halk Sağlığı Genel Müdürlüğü. [COVID-19 (SARS-CoV-2 Infection) Guide]. 2020. [Link]
4. ASRM. American Society for Reproductive Medicine (ASRM) Patient Management and Clinical Recommendations During the Coronavirus (COVID-19) Pandemic. Update \#3 (April 24, 2020 through May 11, 2020) [Link]

5. ESHRE COVID-19 Working Group. ESHRE Guidance on Recommencing ART Treatments. 2020. Date of publication: April 4. Last update: May 5. Published on the ESHRE website.

6. ESHRE Guideline Group on Good Practice in IVF Labs, De los Santos MJ, Apter S, Coticchio G, Debrock S, Lundin K, et al. Revised guidelines for good practice in IVF laboratories (2015). Hum Reprod. 2016;31(4):685-6. [Crossref] [PubMed] 